\title{
A Case of Primary Burkitt Lymphoma of the Bladder
}

\author{
Youssef Bencherki, Oussama El Idrissi Alami, Amine Moataz, Mohammed Dakir, \\ Adil Debbagh, and Rachid Aboutaieb
}

\section{ABSTRACT}

The most frequent primary lymphomas of the bladder are the low-grade Bcell- derived non-Hodgkin's lymphomas of the MALT type, including Burkitt's lymphoma. Primary genitourinary localization of lymphomas is uncommon and, in particular supra vesical development of Burkitt lymphoma. The continuous progress of treatment by chemotherapy has changed the pejorative prognosis of this disease into a good one.

Keywords: Bladder; Burkitt; Lymphoma; Supra-vesical.
Submitted : April 05, 2021

Published : April 26, 2021

ISSN: $2593-8339$

DOI: $10.24018 /$ ejmed.2021.3.2.798

Youssef Bencherki *

Department of urology, Ibn Rochd University Hospital, Casablanca, Morocco.

(e-mail: bencherki.y@outlook.com)

Oussama El Idrissi Alami

Department of urology, Ibn Rochd

University Hospital, Casablanca,

Morocco.

Amine Moataz

Department of urology, Ibn Rochd

University Hospital, Casablanca,

Morocco.

Mohammed Dakir

Department of urology, Ibn Rochd

University Hospital, Casablanca,

Morocco.

Adil Debbagh

Department of urology, Ibn Rochd

University Hospital, Casablanca,

Morocco.

Rachid Aboutaieb

Department of urology, Ibn Rochd

University Hospital, Casablanca,

Morocco.

*Corresponding Author

\section{INTRODUCTION}

Primary genitourinary localization of lymphomas is uncommon and, in particular supra vesical development of Burkitt lymphoma. This lymphoma is one of the most aggressive neoplasms, by its prompt proliferation and his association with a tumor lysis syndrome. The continuous progress of treatment by chemotherapy has changed the pejorative prognosis of this disease into a good one.

\section{CASE REPORT}

We describe a case of 15 years old boy, with no past medical history, who presented with 10 months history of hypogastric mass associated with lower tract symptoms as dysuria and frequency, without any episode of hematuria.

The physical examination revealed a boy with a good performance status, hypogastric mass that measures $10 \mathrm{~cm}$ in its major axis, and bilateral infracentimetrique inguinal lymphadenopathy.

Initial laboratory exams revealed a WBC count of $8420 / \mathrm{mm}^{3}$, hemoglobin count of $12,2 \mathrm{~g} / \mathrm{dl}$, and platelet count of $350000 / \mathrm{mm}^{3}$. Serum chemistry showed a normal level of lactate dehydrogenase, of kidney function, of calcium and of phosphorus. Cytobacteriological examination of the urine did not show microscopic hematuria or bacteriuria.

A computed tomography scan of the abdomen and pelvis was performed, and represented a large mass extend from umbilicus to vesical dome, with an infiltration of the anterior and lateral wall of the bladder. This process had an endo vesical extension, and it is heterogeneously enhanced after injection of the contrast product, thus delimiting areas of necrosis (Fig. 1). 

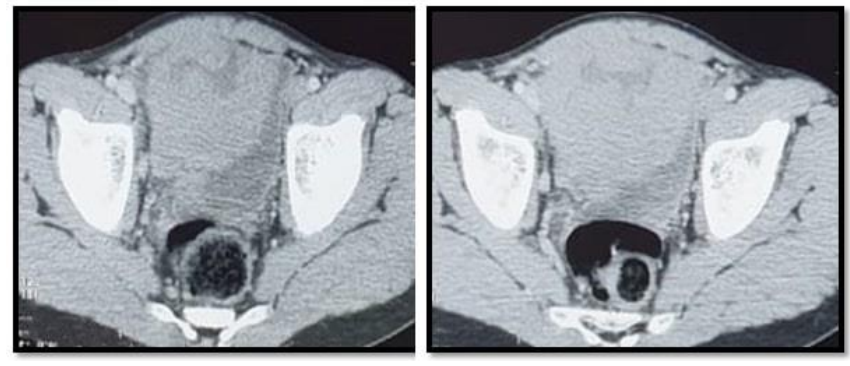

Fig. 1. Computed tomography of the lower abdomen and pelvis demonstrates the urinary bladder thickening of the entire wall.

Cystoscopy was performed, showing a bulge at the level of the dome and on the anterior bladder wall. Biopsies with forceps were performed objectifying a morphological aspect of chronic cystitis without sign of malignancy.

After a multidisciplinary consultation meeting, we decided to do a percutaneous biopsy. Immunohistochemical studies concluded to a Burkitt Lymphoma with a positive CD20, CD3, CD10 and BCL6, whereas BCL2 were negative. 100\% positivity for Ki-67 immunoreactivity was seen (Fig. 2-4).

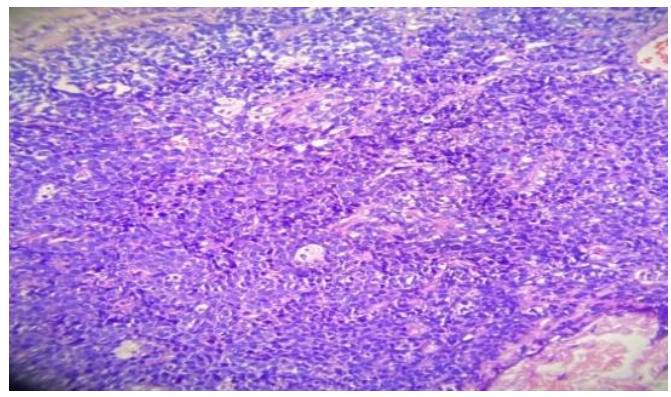

Fig. 2. Anatomopathology

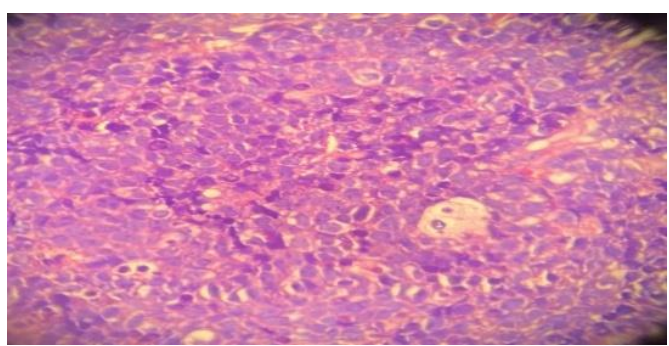

Fig. 3. Anatomopathology.

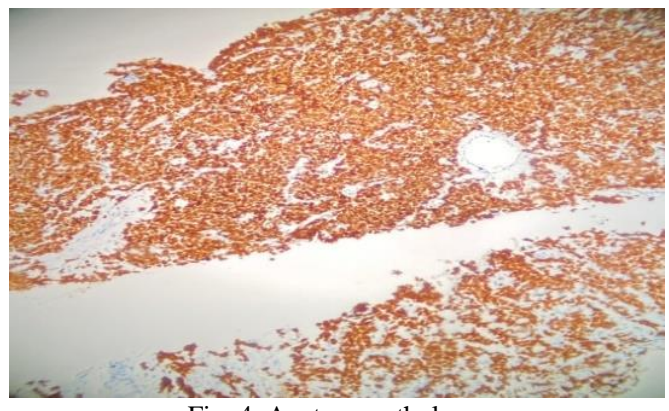

Fig. 4. Anatomopathology.

The patient received chemotherapy, with 1 course of COP and 2 courses of COPADM (Cyclophosphamide, Vincristine, Adriamycin, High dose Methotrexate and Prednisolone). After such a treatment, the patient had no symptoms and a normal physical examination. Moreover, no evidence of disease was found in a new CT scan. Therefore, the patient was in complete response.

\section{DISCUSSION}

Incidental involvement of the genitourinary system by lymphoma is rare. $6-8 \%$ of patients with aggressive lymphoma will manifest urological symptoms from the genitourinary extension [1].

Stomach is the most common sites of primary extranodal lymphoma. The bladder localization is very rare and represented less than $1 \%$ of bladder cancer [2], [3].

Primary bladder lymphoma affects more women than men, especially those who present repeated cystitis. The most common symptom is hematuria.

Radiological demonstration of a hydronephrosis or a mass involving the bladder, as in the present case, is indicative of genitourinary infiltration, but to confirm this infiltration a biopsy is necessary.

Cytological examination of the urine sediment is not only helpful in the diagnosis of renal involvement by a variety of solid tumors and hematological malignancies, but also be valuable in localizing the tumor. In patients with lymphomatous involvement of the bladder or ureter, hematuria marked acute inflammation and benign urothelial cells may be associated with the malignant cells [3]-[5].

The most frequent primary lymphomas of the bladder are the low-grade B-cell- derived non-Hodgkin's lymphomas of the MALT type, including Burkitt's lymphoma. The 3 types of Burkitt lymphoma are endemic or African (most often characterized by jaw involvement), sporadic or non-African (most often intra-abdominal), and immunodeficiency associated.

At a younger age range, we must think about rhabdomyosarcoma as a differential diagnosis, which represents $6 \%$ of tumors in children under fifteen years old.

The treatment of bladder lymphoma is the same as that for nodal lymphomas. Surgery, radiotherapy, and chemotherapy are the options for the primary bladder lymphomas. Surgery can be a solution for the irritative urinary symptoms. In our case we did not performed any surgery, but it is the chemotherapy that helped us improving urinary symptoms.

The treatment strategy for these lymphomas is based on the use of intensive multidrug combinations for short periods. In our case the patient received 1 course of COP and 2 courses of COPADM.

\section{CONCLUSION}

In the presented case the diagnosis of primary bladder lymphoma was made after excluding a systemic hematological malignancy. Malignant lymphoma of the urinary bladder is characterized by a marked preponderance of women who are occasionally affected with chronic cystitis. There is no consensus regarding the treatment of primary lymphoma of the bladder, owing to the lack of large patient series. A variety of therapies, including chemotherapy radiotherapy and surgery, have been favored for primary bladder lymphoma. In the current patient, following four cycles of chemotherapy, the tumor and its related symptoms did not reappear. 


\section{DECLARATION OF COMPETING INTEREST}

The authors state that they do not have competing interests.

\section{AVAILABILITY OF DATA AND MATERIAL}

The datasets in this article are available in the repository of the urology database, CHU Ibn-Rochd Casablanca, upon request, from the corresponding author.

\section{AUTHOR'S CONTRIBUTION}

Dr. Bencherki Youssef and Dr. El Idrissi Alami Oussama analysed and performed the literature research, Pr. Moataz Amine, Pr. Dakir Mohammed, Pr. Debbagh Adil and Pr. Aboutaieb Rachid performed the examination and performed the scientific validation of the manuscript. Bencherki Youssef and El Idrissi Alami Oussama were the major contributors to the writing of the manuscript. All authors read and approved the manuscript.

\section{REFERENCES}

[1] Weimar, G., Culp, D.A., Leoning, S. and Narayana, A. (1981) "Urological involvement in malignant lymphomas", J. Urol. 125, 230231.

[2] Kuhara $\mathrm{H}$ et al. (1990) Primary malignant lymphoma of the urinary bladder. A case report, Acta Pathol Jpn 40: 764-769.

[3] Cheson, B.D., Schumann, G.B. and Johnson, J.L. (1984) "Urinary cytodiagnosis of renal involvement in disseminated histiocytic lymphoma", Acta Cytol. 28, 148-151.

[4] Sano, M.E. and Koprowska, L. (1965) "Primary cytologic diagnosis of a malignant renal lymphoma", Acta Cytol. 9, 194-196.

[5] Prince, J.P., Graham, R.C. and Bernier, G.M. (1974) "Detection of myeloma cells in the urine sediment", Blood 43, 137-143. 